\title{
Zoledronic acid in the management of metastatic bone disease
}

\author{
Thomas J Polascik \\ Vladimir Mouraviev \\ Duke Prostate Center and Division \\ of Urologic Surgery, Duke University \\ Medical Center, Durham, NC, USA
}

\begin{abstract}
Many patients with advanced cancer experience decreased bone strength due to metastatic foci, underlying osteoporosis and/or cancer treatment induced bone loss. The clinical consequences of metastatic disease involving the skeleton are widespread. This review focuses on the efficacy, pharmacology, and safety when using intravenous biphosphonate such a zoledronic acid for cancer bone metastases. Zoledronic acid is the gold standard for the medical management of metastatic bone disease. The indications for treatment include prevention of skeletal relevant events (SRE), osteoporotic complications, and palliation of bone pain, among others. Zoledronic acid is the only bisphosphonate effective in decreasing SREs associated with bone metastases from advanced renal cell carcinoma and prostate cancer. Regarding prostate cancer, zoledronic acid effectively prevents both bone loss in patients with locally advanced disease receiving androgen deprivation therapy and SREs in men with hormone-refractory or hormonesensitive metastatic disease. Zoledronic acid has an acceptable safety profile and tolerability, and has been effective at significantly decreasing the incidence, delaying the onset, and reducing the overall risk of experiencing an SRE compared to placebo. It is the only bisphosphonate currently approved for the prevention and treatment of skeletal complications in patients with bone metastases due to all solid tumors.
\end{abstract}

Keywords: zoledronic acid, metastatic bone disease, osteoporosis, skeletal relevant events, advanced prostate cancer

\section{Introduction to management of metastatic bone disease}

Many patients with advanced cancer experience decreased bone strength due to metastatic foci, underlying osteoporosis, and/or cancer-treatment-induced bone loss. Patients at risk of bone loss include smokers, those with excessive alcohol use, low calcium intake, sedentary lifestyle, and hypogonadal men. Osteoporosis affects approximately 75 million people in Europe, the United States, and Japan (International Osteoporosis Foundation 2007). Although the focus of development of osteoporosis has been associated with aging women, it has only recently been appreciated that men remain at high risk for osteoporosis, as the National Osteoporosis Foundation estimates that 2 million American men have the disease and is increasing. Androgen deprivation therapy, which is commonly employed therapeutically in advanced prostate cancer, increases bone loss and subsequent fracture risk (Daniell et al 1997).

The potential consequences of metastatic cancer affecting the skeleton are multiple. First, it often signals a significant change in quality of life as patients are more likely to develop skeletal fractures, bone pain, hypercalcemia of malignancy, or spinal cord compression. Second, extensive bone metastases accompany poor health and diminished survival (Soloway et al 1988; Sabbatini et al 1999). Third, skeletal-related events (SREs) invariably accompany waning quality of life. Fractures diminish quality of life, and even unrecognized fracture can cause functional impairment, decreased mobility 
or pain. Patients with bone metastases are at increased risk of mortality as the ultimate demise of the patient often is coupled to skeletal complications (Coleman et al 2005).

The more common human cancers metastasizing to bone are breast, prostate, multiple myeloma, and kidney. The incidence of multiple myeloma in the United States is estimated at 15,000 annually, and these malignant plasma cells usually attack bone structure causing osteolytic lesions. In regards to breast cancer, bone is the most common site of distant disease recurrence and is estimated to affect up to $75 \%$ of women with advanced breast cancer (Coleman et al 2001). Of those with bone metastasis, two-thirds will develop a SRE (Plunkett et al 2000). The median survival with advanced breast cancer is about 18-26 months after diagnosis of bone metastasis, placing women at risk of skeletal complications (Coleman et al 2001). Approximately one-third of patients with advanced renal cell carcinoma develop bone metastases. However once this occurs the 2-year survival rate decreases to $10 \%-20 \%$ with a median survival of 1 year (Flanigan et al 2003; Martel et al 2003). The natural history of untreated patients with prostate cancer and bone metastases show that almost $50 \%$ had experienced at least one SRE by 2 years, the majority of which comprised a pathologic fracture(s) or need for palliative radiation (Saad et al 2004). Greater than $80 \%$ of men dying of prostate cancer have autopsy-proven bone metastases (Harada et al 1992).

In contrast to bone lesions secondary to metastatic breast cancer, the majority of lesions from metastatic prostate cancer are osteoblastic. Interestingly, the dysregulated process of bone turnover when affected by cancer invasion also includes increased osteolysis (Garnero et al 2000). Since the process of bone homeostasis is impaired, the new bone deposited under the influence of metastatic cancer cells is poorly mineralized with less integrity. This process increases the risk of a subsequent SRE. Most bone metastases due to renal cell carcinoma are predominantly osteolytic. Of those patients with renal cell carcinoma with bone metastases, approximately one-third of patients develop hypercalcemia of malignancy, half developed long-bone fracture, and the majority required palliative radiation for pain (Zekri et al 2001; Rosen et al 2004a).

Regarding advanced prostate cancer, many men with bone metastases are treated with androgen deprivation therapy which represents the standard of care for metastatic disease. Older age, long duration of androgen deprivation therapy, and low body mass index are risk factors for increased bone loss during androgen deprivation therapy in men with locally advanced disease. Patients with prostate cancer receiving androgen deprivation therapy are at risk of skeletal complications, regardless of disease stage.

The clinical consequences of metastatic disease involving the skeleton are widespread. Of all the bisphosphonate agents available, zoledronic acid is the only agent demonstrated to effectively reduce SREs and delay their onset in patients with advanced renal or prostate cancer metastatic to bone. This review will discuss the efficacy, pharmacology, and safety when using zoledronic acid to treat metastatic disease of the bone.

\section{Pharmacology of zoledronic acid}

Normal bone turnover involves a complex interaction between bone breakdown and bone turnover that is typically in equilibrium. Osteoclasts decompose bone matrix and mineral, allowing mononuclear cells to prepare bone surface for osteoblasts. In turn, osteoblasts lay down new matrix and bone substance. Estrogens and androgens help provide the milieu to maintain normal bone turnover. During the process of metastases, cancer cells create dysregulated bone turnover, with varying degrees of impaired osteolysis and bone synthesis seen depending on the type of cancer. For example, mixed osteoblastic and osteolytic metastases are commonly found in both prostate and breast cancer.

Bisphosphonates are pyrophosphate analogues that bind to hydroxyapatite, accumulate in bone and inhibit osteoclastic activity. As a class, bisphosphonates have several modes of action on osteoclasts. They preferentially bind to bone surfaces undergoing active remodeling. These agents are selectively adsorbed into mineral surfaces on bone that are surrounded by osteoclasts and are then released, whereby they are internalized by and disrupt the bone-resorbing action of osteoclasts (Lipton 2004). Osteoclast maturation is inhibited and apoptosis is induced. Bisphosphonates suppress osteoclast activity and inhibit osteoclast recruitment to sites of bone resorption. Finally, they decrease bone resorbing growth factor and cytokine production. There is some evidence that bisphosphonates may also inhibit cancer cell-bone matrix binding, inhibiting invasion of cancer into the bone (Boissier et al 1997, 2000).

Zoledronic acid is a third generation nitrogen-containing bisphosphonate. Its core bisphosphonate moiety attaches to bone while its imidazole ring containing two nitrogen atoms confirms its potency. Zoledronic acid is approximately 100-fold more potent than pamidronate (Green et al 1994). Bisphosphonates have low intestinal absorption and can cause esophageal mucosal irritation if taken orally. For this 
reason, the formulated intravenous product is well absorbed and remains active in bone for some time.

\section{Efficacy in metastatic disease}

The propensity of cancer to metastasize to bone is governed by ligand-receptor interactions between the host site and the cancer cell type. Tumor cell surface adhesion molecules, such as the chemokine receptor CXCR4-stromal derived factor (SDR-1) interaction, the integrins, and the matrix metalloproteinases are thought to mediate skeletal targeting.

Zoledronic acid is the current standard of care for treating bone metastases from hormone-refractory prostate cancer, advanced breast cancer, stage III multiple myeloma and advanced renal cancer. In advanced breast cancer, randomized trials have demonstrated zoledronic acid to significantly delay the onset and decrease the incidence of SREs when used in conjunction with chemotherapy or hormonal therapy (Kristensen et al 1999; Lipton et al 2000; Body et al 2003, 2004; Rosen et al 2004b). In a randomized, double-blind, phase III study, 1640 patients with breast cancer or multiple myeloma and at least one bone lesion were relegated to zoledronic acid or palmidronate for a 2-year period. Overall, zoledronic acid was superior to palmidronate, the prior standard of care agent, in decreasing overall risk of developing SREs by $16 \%$ at 2 years (Rosen et al 2001). Of those patients with advanced breast cancer and a minimum of one osteolytic lesion, the median time to first SRE was significantly longer when administered zoledronic acid compared to pamidronate (310 vs 174 days, $p=0.013$ ). Zoledronic acid was significantly more effective than palmidronate by decreasing the risk of SREs by an additional $20 \%$ compared to palmidronate and by an additional $30 \%$ in those receiving hormonal therapy (Rosen et al 2001). In a Japanese randomized trial of 228 breast cancer patients with bone metastases, zoledronic acid treated women showed a significant reduction of $39 \%$ in SREs $(\mathrm{p}=0.027)$ when compared to placebo (Kohno et al 2005). Zoledronic acid also demonstrated significantly longer time to first skeletal complication (median not reached vs 364 days placebo, $\mathrm{p}=0.007$ ).

The efficacy of treating skeletal complications of advanced renal cell carcinoma was demonstrated in a subset of 46 kidney cancer patients in a large phase III placebocontrolled trial also involving lung cancer and other solid tumors (Rosen et al 2004b). In this study, most patients (81\% zoledronic acid vs $95 \%$ placebo) had already experienced a SRE before entry. Despite this, zoledronic acid significantly delayed the time to subsequent SRE in the study by nearly 12 months compared to placebo, and significantly reduced the incidence of SREs. Overall the risk of developing a SRE in renal cell carcinoma patients with bone metastases was reduced by $58 \%$ when compared to placebo.

In a randomized, double blind, placebo controlled study of 643 men with metastatic hormone-refractory prostate cancer, zoledronic acid (4 mg IV q $3 \mathrm{wk}$ ) significantly reduced the percentage of patients experiencing SREs compared with placebo at $15(33.2 \%$ vs $44.2 \%, \mathrm{p}=0.02)$ and $24(38 \%$ vs $49 \%, p=0.028$ ) months (Saad et al 2004). The median time to the onset of the first SRE was delayed by more than 5 months (488 vs 321 days; $\mathrm{p}=0.009$ ) (Saad et al 2002, 2004). Zoledronic acid decreased the overall risk of skeletal complications by $36 \%$ at 24 months $(p=0.002)$ (Saad et al 2004). Although study patients reported increasing bone pain throughout the clinical trial, changes in the Brief Pain Index (BPI) score were significantly lower in patients receiving zoledronic acid compared to placebo ( $\mathrm{p}<0.05$ by 21 months) (Saad et al 2004). This suggests a durable reduction in bone pain in zoledronic acid-treated patients with metastatic prostate cancer.

Significant and sustained reductions in bone pain and related complications were achieved using intermittent zoledronic acid for as long as 2 years (Saad et al 2002, 2004). In comparison, studies evaluating the role of other bisphosphonates to reduce bone pain and SREs were more modest. For example, pamidronate, a second generation bisphosphonate, did not significantly diminish the incidence of SREs or bone pain in a combined analysis of two randomized, multicenter, double-blind, placebo-controlled trials (Lipton 2004; Small et al 2003). In patients with metastatic prostate cancer, the first-generation (non-nitrogen-containing) bisphosphonates etidronate and clodronate only provided marginal short-term reductions in pain and analgesic use but did not demonstrate overall clinical benefit (Higano et al 2004; Lipton 2004). A similar trend was seen in a phase II trial evaluating the palliative benefit of second-line zoledronic acid in breast cancer patients with either a SRE or progressive bone metastases despite first-line bisphosphonate therapy. This study of 31 women initially treated with pamidronate or clodronate demonstrated that significant improvements in pain control and bone turnover markers can be achieved when rescued with the more potent bisphosphonate zoledronic acid (Clemons et al 2007). In another study comparing zoledronic acid to pamidronate in patients with bone metastases from breast cancer or multiple myeloma, zoledronic acid significantly decreased the requirement for palliative radiation for bone pain compared to palmidronate ( $19 \mathrm{vs} 24 \%$ for palmidronate, $\mathrm{p}=0.037)$ (Rosen et al 2003). 
Routine use of androgen deprivation therapy as a standard of care to treat advanced prostate cancer leads to significant bone loss and osteoporosis that may increase the risk of skeletal complications especially when compounded by bone metastases. Androgen deprivation therapy is being employed earlier in the stage of disease, such as biochemical relapse without metastatic disease, and is also continued in men who develop hormone-refractory disease. Thus, men in general are beginning hormonal manipulation earlier in the course of disease and remaining on it longer. It has been shown that androgen-deprivation associated bone loss in men is greater than that observed in postmenopausal women (Higano et al 2003). Thus long-term treatment with androgen starvation has long-term clinical implications. In men receiving androgen deprivation therapy for advanced prostate cancer, bone mineral density is decreased by $0.6 \%-5.3 \%$ annually and $2.3 \%-6.6 \%$ in those with metastatic disease, exceeding by 5 to 10 -fold the normal bone loss rates compared with healthy men and prostate cancer patients not treated with hormones (Smith et al 2001; Smith et al 2003; Higano 2004; Casey et al 2006; Israeli et al 2006; Michaelson et al 2006; Ryan et al 2006). Thus, use of androgen deprivation increases the chance of fracture by $7 \%-45 \%$ compared with similar men not under hormonal therapy (Shahinian et al 2005; Smith et al 2005, 2006). The duration of androgen deprivation therapy correlates with risk of hip fracture: $20 \%$ increased risk in the first 3 years, $45 \%$ during years $3-5$ and a $95 \%$ increase in risk if treated with androgen withdrawal for greater than 5 years (Ross and Small 2002).

Zoledronic acid has been shown to reduce metastasisrelated bone complications in prostate cancer patients with progressive disease after treatment of at least one form of androgen deprivation therapy (Saad et al 2002, 2004; Casey et al 2006). In prostate cancer patients with hormonesensitive skeletal metastatic disease receiving androgen deprivation therapy, zoledronic acid significantly increased bone mineral density and the median time to first SRE was not reached (Polascik et al 2005). This suggests that zoledronic acid prevents bone complications in men with both hormonesensitive and hormone-resistant metastatic prostate cancer. The National Comprehensive Cancer Network Guidelines on Prostate Cancer state that early bisphosphonate therapy of all men with prostate cancer treated with androgen deprivation therapy may prevent androgen-deprivation and/or bone metastasis related morbidity and improve quality of life (NCCN guidelines).

At the present time, zoledronic acid is the only bisphosphonate that has been demonstrated to decrease SREs in prostate cancer, breast and renal cell carcinoma patients. Zoledronic acid has been shown to be effective for treatment of bone metastases regardless of whether the bone lesions are predominantly osteolytic or osteoblastic. Patients are more likely to experience additional skeletal complications once the initial SRE has occurred (Saad et al 2004). For this reason it is recommended to continue zoledronic acid as a means to prevent further fracture or SREs. Currently, studies are being undertaken in patients with advanced cancer to address the use of bisphosphonates for the prevention of bone metastases.

\section{Safety and tolerability}

Zoledronic acid can be administered safely with good tolerability. It is recommended to administer the medication intravenously over at least 15 minutes. Patients must be adequately hydrated and a serum creatinine level should be assessed prior to administration. It is recommended that patients take vitamin $\mathrm{D}$ and supplemental calcium daily.

The incidence of adverse events occurring in greater than $10 \%$ of patients on zoledronic acid include back pain (12\%), nausea (14\%), fatigue (14\%) or arthralgia (20\%) (Zometa $^{\circledR}$ [package insert] 2006). Fever, vertigo, asthenia, and renal tubular necrosis have been observed at a lower incidence. Flu-like symptoms, characterized by fever, myalgia, and/or fatigue, have been observed in up to $20 \%$ of patients but are usually associated with the first administration. Serum calcium levels should also be periodically monitored; however, hypocalcemia is rarely observed in patients taking calcium supplementation.

Before commencing bisphosphonate therapy, patients should be evaluated for renal insufficiency. A calculated creatinine clearance is used for dose adjustment for patients with mild to moderate renal insufficiency. Dose adjustments are required for those with a baseline creatinine clearance less than $60 \mathrm{~mL} /$ minute (Table 1). Clinical trials have demonstrated that renal safety of zoledronic acid over time was comparable to placebo (Saad et al 2002). Zoledronic acid did not impair renal function in patients with renal cell carcinoma, a group that is believed to be at a higher risk for such occurrence (Rosen et al 2004b). The $4 \mathrm{mg}$ intravenous dose has been shown to have a much lower effect on renal toxicity than the $8 \mathrm{mg}$ dose used in early studies. In clinical trials evaluating zoledronic acid (4 mg IV q $3 \mathrm{mo}$ ) in locally advanced prostate cancer, serum creatinine levels significantly increased in fewer than $1 \%$ of patients (Smith et al 2003; Casey et al 2006; Israeli et al 2006; Michaelson et al 2006; Ryan et al 2006). These studies' results suggest 
Table I Dosing zoledronic acid with renal insufficiency

\begin{tabular}{lll}
\hline $\begin{array}{l}\text { Creatinine clearance } \\
(\mathbf{m L} / \mathbf{m i n})\end{array}$ & $\begin{array}{l}\text { Recommended } \\
\text { dose }(\mathbf{m g})\end{array}$ & $\begin{array}{l}\text { Amount to be with- } \\
\text { drawn from vial }(\mathbf{m L})\end{array}$ \\
\hline$>60$ & 4.0 & 5 \\
$50-60$ & 3.5 & 4.4 \\
$40-49$ & 3.3 & 4.1 \\
$30-39$ & 3.0 & 3.8 \\
\hline
\end{tabular}

that renal impairment may be more common in patients with shorter infusion times (eg, 5 vs 15 minutes) and dosage intervals (eg, q 3 weeks vs q 3 months). Treatment is withheld if further renal impairment occurs and may be resumed when serum creatinine levels return to $10 \%$ of baseline. Renal dysfunction can be prevented and managed with appropriate monitoring and intervention.

Osteonecrosis of the jaw (ONJ) is a complication that has received much attention since 2003 . This condition is defined as avascular necrosis resulting from impaired blood supply to bone, usually unassociated with infection. It is clinically diagnosed by exposed bone in the maxillofacial area, poor wound healing, and pain. The etiology and pathogenesis remain unclear as of this writing; however, is likely to be multifactorial. Risk factors for ONJ include oral infection, inflammation, or oral surgery, poor dental hygiene, alcohol abuse, or concomitant radiation or chemotherapy. Relatively few cases have been reported in patients receiving intravenous bisphosphonates. Although ONJ can be diagnosed by the lack of healing following 6 weeks of appropriate dental care in the absence of osteoradionecrosis or metastatic disease to the jaw, no uniform diagnostic criteria have been established.

ONJ tends to occur with long exposure to bisphosphonates. In largest retrospective study from MD Anderson Cancer Center including 4000 patients who received intravenous biphosponates the frequency of ONJ was $0.73 \%$ overall, $1.2 \%$ in breast cancer and $2.4 \%$ in MM (Hoff et al 2006). Patients with ONJ have had longer duration of disease and received greater doses of biphosponate therapy than patients without ONJ.

Prevention and good oral hygiene are important strategies to reduce the chance of experiencing ONJ. It is recommended that patients have a dental examination and any dental procedures completed prior to commencing bisphosphonate therapy. While on bisphosphonate medication, patients should avoid dental surgery, maintain good hygiene, and have a routine dental exam every 6 months. For those requiring dental surgery, no data exist as to whether discontinuing bisphosphonates diminishes the risk of ONJ. If ONJ is contracted, management is conservative, using appropriate antibiotics, oral rinses, and pain control. The prevalence and guidelines for preventing and managing ONJ need to be more fully characterized.

Since bisphonates may allay pain in addition to stabilizing bone density, patients often combine zoledronic acid with other pain therapies, such as opioids, radiation therapy, and/or chemotherapy (Saad et al 2002). Nonsteroidal anti-inflammatory medication should only be used with caution due to the risk of renal impairment (Berenson 2005). Zoledronic acid generally decreases the need for additional therapies to treat pain over time (Saad et al 2002, 2004). Bisphosphonates are generally well tolerated when employed with cytotoxic chemotherapy agents.

The long-term safety of bisphosphonates beyond 2 years has not been published to date. In patients with metastatic prostate cancer, 2 years of zoledronic acid therapy was well tolerated (Saad et al 2004). The mean survival of patients with advanced cancer and bone metastases usually does not extend beyond this interval.

\section{Patient perspectives}

Quality of life remains the main focus from the patient perspective. The major problem in many advanced cancers, particularly prostate, breast, and renal cancer, is related to the bone. Up to $70 \%$ of patients with advanced prostate cancer have or will develop bone metastases. As such, these patients are at high risk for skeletal complications. Any skeletal-related event, defined as pathologic fracture, spinal cord compression, change in antineoplastic therapy (due to pain), need for radiation therapy or surgery due to bone disease, or development of hypercalcemia of malignancy, will impact quality of life due to both the event itself and also the need for further therapy.

Bone-related complications from advanced cancer impact quality of life by three common mechanisms: bone pain, impaired mobility, and pathologic fracture. Fracture risk increases with reduced bone mineral density (Faulkner 2000; Cummings et al 2002). Regarding the latter, bone fracture negatively correlates with patient survival (Oefelein et al 2002). Pathologic fracture has been demonstrated to increase risk of death by $29 \%$ in men with hormone-refractory prostate cancer (Saad et al 2005). Most osteoporotic fractures do not spontaneously heal and require further treatment. With advanced cancer involving bone metastases, patients are not uncommonly dependent of strong narcotics to treat the debilitating pain of bone lesions or may require palliative radiation or systemic radiopharmaceuticals. 
A FACT-G (Functional Assessment of Cancer TherapyGeneral) study demonstrated what is already suspected - that bone complications affect quality of life with resulting significant reduction in emotional, physical, and functional domains (Weinfurt et al 2002). In a study of 248 men with hormone-resistant prostate cancer metastatic to bone with greater than $1 \mathrm{SRE}$, health-related quality of life was measured with EURO-EQ-5D and FACT-G questionnaires along with bone pain index interference and intensity scales. Every SRE experienced resulted in reductions in multiple quality of life domains, and placed greater burden on both the patient and the caregiver (Weinfurt et al 2005).

Similarly, treatment with zoledronic acid has also benefited women with advanced breast cancer. In a health-related quality of life study among patients with breast cancer receiving zoledronic acid for metastatic bone disease, investigators concluded that prevention of further SRE resulted in an overall increase in health-related quality of life (Weinfurt et al 2004). In a study of 101 women with breast cancer and at least 1 bone metastasis treated with hormonal therapy, the European Organisation for Research and Treatment of Cancer Quality of Life Core Questionnaire 30 (EORTC QLQ-C30) and the Brief Pain Inventory (BPI) were used to assess potential benefits of zoledronic acid. At end of study, the BPI demonstrated significant reductions in worst pain $(p=0.008)$, average pain within the last week $(\mathrm{p}=0.039)$ and interference with general activity $(\mathrm{p}=0.012)$. Overall health status demonstrated a significant median improvement of $8.3 \%(p=0.013)$ and emotional, social, and physical functioning also showed significant overall improvement $(\mathrm{p}=0.013,0.005$, and 0.043 , respectively) at end of study (Wardley et al 2005).

Bone complications resulting from bone metastases increase health care costs. These additional costs affect both the patient and the health care system. An insurancebased study of US healthcare claims between 1994 and 2002 showed a difference in total medical costs of over US\$20,000 per prostate cancer patient with the presence of bone metastases compared with those without skeletal lesions (McKiernan et al 2004).

In addition, patients should take on the responsibility of active participation in their care when possible. Since many patients with advanced cancer have or are at risk of osteoporosis, it is recommended that patients improve their lifestyle by establishing a routine weight-resistance exercise routine, limiting alcohol intake, and taking vitamin D and calcium supplements. It is noted that vitamin D and calcium supplementation alone were not sufficient to prevent bone loss in the placebo arm of men with advanced prostate cancer requiring androgen deprivation therapy (Smith et al 2003). For those who use tobacco products, smoking cessation is highly recommended. These modifications aid in decreasing bone loss in otherwise healthy people at risk of osteoporosis (Higano 2004; Wegner 2006).

\section{Conclusions}

Zoledronic acid is the gold standard for the medical management of metastatic bone disease. The indications for zoledronic acid include prevention of skeletal events, osteoporotic complications, and palliation of bone pain, among others. Zoledronic acid is the only bisphosphonate effective in decreasing SREs associated with bone metastases from advanced renal cell carcinoma and prostate cancer. Regarding prostate cancer, zoledronic acid effectively prevents both bone loss in patients with locally advanced disease receiving androgen deprivation therapy and SREs in men with hormone-refractory or hormone-sensitive metastatic disease. Zoledronic acid has an acceptable safety profile and tolerability, and has been effective at significantly decreasing the incidence, delaying the onset, and reducing the overall risk of experiencing an SRE compared with placebo. It is the only bisphosphonate currently approved for the prevention and treatment of skeletal complications in patients with bone metastases due to all solid tumors.

\section{References}

Berenson JR. 2005. Recommendations for zoledronic acid treatment of patients with bone metastases. Oncologist, 10:52-62.

Body JJ, Diel IJ, Lichinitzer M, et al. 2004. Oral ibandronate reduces the risk of skeletal complications in breast cancer patients with metastatic bone disease: results from two randomized, placebo controlled phase III studies. Br J Cancer, 90:1133-7.

Body JJ, Diel IJ, Lichinitser MR et al. On behalf of the MF 4625 study group. 2003. Intravenous ibandronate reduces the incidence of skeletal complications in patients with breast cancer and bone metastases. Ann Oncol, 14:1399-405.

Boissier S, Ferreras M, Peyruchaud O, et al. 2000. Bisphosphonates inhibit breast and prostate carcinoma cell invasion, an early event in the formation of bone metastases. Cancer Res, 60:2949-54.

Boissier S, Magnetto S, Frappart L, et al. 1997. Bisphosphonates inhibit prostate and breast carcinoma cell adhesion to unmineralized and mineralized bone extracellular matrices. Cancer Res, 57:3890-4.

Casey R, Love W, Mendoza C, et al. 2006. Zoledronic acid reduces bone loss in men with prostate cancer undergoing androgen deprivation therapy. Presented at 2006 Multidisciplinary Prostate Cancer Symposium. February 24-26, 2006; San Francisco, Calif. Abstract \#184.

Clemons MJ, Dranitsaris G, Ooi WS, et al. 2006. Phase II trial evaluating the palliative benefit of second-line zoledronic acid in breast cancer patients with either a skeletal-related event for progressive bone metastases despite first-line bisphosphonate therapy. J Clin Oncol, 24:4895-900.

Coleman RE, Major P, Lipton A, et al. 2005. Predictive value of bone resorption and formation markers in cancer patients with bone metastases receiving the bisphosphonate zoledronic acid. J Clin Oncol, 23:4925-35. 
Cummings SR, Bates D, Black DM. 2002. Clinical use of bone densitometry: scientific review. JAMA, 288:1889-97.

Daniell HW. 1997. Osteoporosis after orchiectomy for prostate cancer. J Urol, 157:439-44.

Faulkner KG. 2000. Bone matters: are density increases necessary to reduce fracture risk? J Bone Miner Res, 15:183-7.

Flanigan RC, Campbell SC, Clark JI, et al. 2003. Metastatic renal cell carcinoma. Curr Treat Options Oncol, 4:385-90.

Garnero P, Buchs N, Zekri J, et al. 2000. Markers of bone turnover for the management of patients with bone metastases form prostate cancer. Br J Cancer, 82:858-64.

Green JR, Muller K, Jaeggi KA. 1994. Preclinical pharmacology of CGP 42 ' 446 , a new, potent, heterocyclic bisphosphonate compound. J Bone Miner Res, 9:745-51.

Harada M, Iida M, Yamaguchi M, et al. 1992. Analysis of bone metastasis of prostatic adenocarcinoma in 137 autopsy cases. Adv Exp Med Biol, $324: 173-82$.

Higano CS. 2004. Understanding treatments for bone loss and bone metastases in patients with prostate cancer: a practical review and guide for the clinician. Urol Clin N Am, 31:331-52.

Higano CS. 2003. Bone loss and the evolving role of bisphosphonate therapy in prostate cancer. Urol Oncol, 21:392-8.

Hoff AO, Toth B, Altundag K, et al. 2006 Osteonecrosis of the Jaw in Patients Receiving Intravenous Bisphosphonate Therapy. JCO, 24(suppl 1):8528.

International Osteoporosis Foundation. The facts about osteoporosis and its impact [online]. Accessed January 10, 2007. URL: http://www. iofbonehealth.org/facts.

Israeli RS, Rosenberg S, Saltzstein D, et al. 2006. Bone mineral density in patients with prostate cancer undergoing initial androgen-deprivation therapy. J Urol, 175(Suppl 4):134. Abstract 410.

Kohno N, Aogi K, Minami H, et al. 2005. Zoledronic acid significantly reduces skeletal complications compared with placebo in Japanese women with bone metastases from breast cancer: a randomized, placebo-controlled trial. J Clin Oncol, 23:3314-21.

Kristensen B, Ejlertsen B, Groenvold M, et al. 1999. Oral clodronate in breast cancer patients with bone metastases: a randomized study. J Intern Med, 246:67-74.

Lipton A. 2004. Pathophysiology of bone metastases: how this knowledge may lead to therapeutic intervention. J Support Oncol, 2:205-213.

Lipton A, Theriault R, Hortobagyi G, et al. 2000. Pamidronate prevents skeletal complications and is effective palliative treatment in women with breast carcinoma and osteolytic bone metastases: long-term follow-up of two randomized controlled trials. Cancer, 88:1082-90.

McKiernan JM, Delea TE, Liss M, et al. 2004. Impact of skeletal complications on total medical care costs in prostate cancer patients with bone metastases. ProcAm Soc Clin Oncol, 23:531, A6057.

Martel CL, Lara PN. 2003. Renal cell carcinoma: current status and future directions. Crit Rev Oncol Hematol, 45:177-90.

Michaelson MD, Lee H, Kaufman DS, et al. 2006. Annual zoledronic acid to prevent gonadotropin-releasing hormone agonist-induced bone loss in men with prostate cancer: a randomized placebo-controlled trial. J Clin Oncol, 24(18S pt 1):220s. Abstract \#4515.

Oefelein MG, Ricchiuti V, Conrad W, et al. 2002. Skeletal fractures negatively correlate with overall survival in men with prostate cancer. J Urol, 168:1005-7.

Polascik TJ, Given RW, Metzer C, et al. 2005. Open-label trial evaluating the safety and efficacy of zoledronic acid in preventing bone loss in patients with hormone-sensitive prostate cancer and bone metastases. Urology, 66:1054-9.

Plunkett TA, Smith P, Rubens RD. 2000. Risk of complications from bone metastases in breast cancer: implications for management. Eur J Cancer, 36:476-82.

Rosen LS, Gordon DH, Dugan W, et al. 2004a. Zoledronic acid is superior to pamidronate for the treatment of bone metastases in breast carcinoma patients with at least one osteolytic lesion. Cancer, 100:36-43.
Rosen LS, Gordon D, Kaminski M, et al. 2001. Zoledronic acid versus palmidronate in the treatment of skeletal metastases in patients with breast cancer or osteolytic lesions of multiple myeloma: a phase III, double-blind, comparative trial. Cancer, J 7:377-87.

Rosen LS, Gordon D, Kaminski M, et al. 2003. Long-term efficacy and safety of zoledronic acid compared with pamidronate disodium in the treatment of skeletal complications in patients with advanced multiple myeloma or breast carcinoma: a randomized, double-blind, multicenter, comparative trial. Cancer, 98:1735-44.

Rosen LS, Gordon D, Tchekmedyian NS, et al. 2004b. Long-term efficacy and safety of zoledronic acid in the treatment of skeletal metastases in patients with non-small cell lung carcinoma and other solid tumors: a randomized, phase III double-blind, placebo-controlled trial. Cancer, 100:2613-21.

Ross RW, Small EJ. 2002. Osteoporosis in men treated with androgen deprivation therapy for prostate cancer. J Urol, 167:1952-6.

Ryan CW, Huo D, Demers LM, et al. 2006. Zoledronic acid initiated during the first year of androgen deprivation therapy increases bone mineral density in patients with prostate cancer. $J$ Urol, 176:972-8.

Saad F, Gleason DM, Murray R, et al. 2002. A randomized, placebocontrolled trial of zoledronic acid in patients with hormone-refractory metastatic prostate carcinoma. J Natl Cancer Inst, 94:1458-68.

Saad F, Gleason DM, Murray R, et al. 2004. Long-term efficacy of zoledronic acid for the prevention of skeletal complications in patients with metastatic hormone-refractory prostate cancer. $J$ Natl Cancer Inst, 96:879-82.

Saad F, Lipton A. 2005. Zoledronic acid is effective in preventing and delaying skeletal events in patients with bone metastases secondary to genitourinary cancers. BJU Internat, 96:964-9.

Sabbatini P, Larson SM, Kremer A, et al. 1999. Prognostic significance of extent of disease in bone in patients with androgen-independent prostate cancer. J Clin Oncol, 17:948-57.

Shahinian VB, Kuo YF, Freeman JL, et al. 2005. Risk of fracture after androgen deprivation for prostate cancer. $N$ Engl J Med, 352:154-64.

Small EJ, Smith MR, Seaman JJ, et al. 2003. Combined analysis of two multicenter, randomized, placebo-controlled studies of pamidronate disodium for the palliation of bone pain in men with metastatic prostate cancer. J Clin Oncol, 21:427784.

Smith MR, Boyce SP, Moyneur E, et al. 2006. Risk of clinical fractures after gonadotropin-releasing hormone agonist therapy for prostate cancer. J Urol, 175:136-9.

Smith MR, Eastham J, Gleason DM, et al. 2003. Randomized controlled trial of zoledronic acid to prevent bone loss in men receiving androgen deprivation therapy for nonmetastatic prostate cancer. $J$ Urol, 169:2008-12.

Smith MR, Lee WC, Brandman J, et al. 2005. Gonadotropin-releasing hormone agonists and fracture risk: a claims-based cohort study of men with nonmetastatic prostate cancer. J Clin Oncol, 23:7897-903.

Smith MR, McGovern FJ, Zietman AL, et al. 2001. Pamidronate to prevent bone loss during androgen-deprivation therapy for prostate cancer. N Engl J Med, 345:948-55.

Soloway MS, Hardeman SW, Hickey D, et al. 1988. Stratification of patients with metastatic prostate cancer based on extent of disease on initial bone scan. Cancer, 61:195-202.

The NCCN (2.2005) Prostate Cancer Clinical Practice Guidelines in

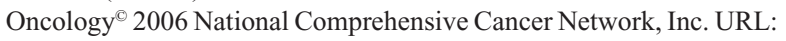
http://www.nccn.org.

Wardley A, Davidson N, Barrett-Lee P, et al. 2005. Zoledronic acid significantly improves pain scores and quality of life in breast cancer patients with bone metastases: a randomized, crossover study of community vs hospital bisphosphonate administration. Br J Cancer, 92:1869-76.

Wegner H. 2006. Clinical experience with zoledronic acid: a urologist's perspective. Eur Urol Suppl, 5:551-2.

Weinfurt KP, Castel LD, Li Y, et al. 2004. Health-related quality of life among patients with breast cancer receiving zoledronic acid or palmidronate disodium for metastatic bone lesions. Medical Care, 42:164-75. 
Weinfurt KP, Li Y, Castel LD, et al. 2002. The impact of skeletal-related events on health-related quality of life of patients with metastatic prostate cancer. Ann Oncol, 13:180.

Weinfurt KP, Li Y, Castel LD, et al. 2005. The significance of skeletalrelated events for the health-related quality of life of patients with metastatic prostate cancer. Ann Oncol, 16:579-84.

Zekri J, Ahmed N, Coleman RE, et al. 2001. The skeletal metastatic compications of renal cell carcinoma. Int J Oncol, 19:379-82.

Zometa $^{\circledR}$ [package insert]. 2006. East Hanover, NJ; Novartis Pharmaceuticals Corporation. 